\title{
Causes of misdiagnosis in assessing tubal patency by transvaginal real-time three-dimensional hysterosalpingo-contrast sonography
}

Na Liang 1

DQing-Qing Wu ${ }^{1}$

(iD) Jing-Hua Li ${ }^{1}$

(iD) Fena-Yun Gao ${ }^{1}$

(iD) Fu-Li Sun ${ }^{1}$

(iD) Cui-Xia Guo ${ }^{1}$

1. Department of Ultrasound, Beijing Obstetrics and Gynecology Hospital, Capital Medical University, Beijing 100026, China

\section{SUMMARY}

OBJECTIVE: This study aims to investigate the causes of misdiagnosis in assessing tubal patency by transvaginal real-time three-dimensional hysterosalpingo-contrast sonography (TVS RT-3D-HyCoSy), in order to improve the diagnostic efficiency of TVS RT-3D-HyCoSy.

METHODS: A total of 162 oviducts of 83 infertility patients were examined by TVS RT-3D-HyCoSy. These results were compared with the gold standard for laparoscopic dye studies, and the misdiagnosed cases were analyzed.

RESULTS: TVS RT-3D-HyCoSy revealed that 68 oviducts were unobstructed and 94 obstructed. The results for the 144 oviducts were in line with the gold standard, while those for 18 oviducts were not. The accuracy rate of the TVS RT-3D-HyCoSy was $88.9 \%$, and the misdiagnosis rate was $11.1 \%$. The main causes of misdiagnosis included contrast medium countercurrent and diffusion, oviduct spasm, abnormal shape or position of the oviduct, pelvic adhesion, and poor imaging operation.

CONCLUSION: TVS RT-3D-HyCoSy can well-evaluate tubal patency, and understand and improve the cause of misdiagnosis. Furthermore, the diagnostic efficiency of TVS RT-3D-HyCoSy can still be further improved.

KEYWORDS: Hysterosalpingography. Infertility. Diagnostic Techniques, Obstetrical and Gynecological. Diagnostic Errors.

\section{INTRODUCTION}

Transvaginal real-time three-dimensional hysterosalpingo-contrast sonography (TVS RT-3D-HyCoSy) has the advantages of non-radiation and non-invasion. At present, it could be easily accepted by infertility patients, when compared to traditional lipiodol oviduct radiography, and has become a new method to check for tubal patency. ${ }^{1}$ In recent years, several multi-center studies at home and abroad have revealed that, compared with the gold standard, TVS RT-3D-HyCoSy has better consistency in the diagnosis of tubal patency ${ }^{2-4}$. However, the misdiagnosis rate continues to bring some difficulties in clinic practice. ${ }^{5}$ In the present study, misdiagnosed cases of TVS RT-3D-HyCoSy were analyzed in order to determine the causes of misdiagnosis, and improve the diagnostic efficiency of TVS RT-3D-HyCoSy in evaluating tubal patency. 


\section{METHODS}

\section{Clinical data}

A total of 83 infertility patients (162 oviducts), who underwent TVS RT-3D-HyCoSy from April 2015 to April 2017 in our hospital, were included in the present study. Among these patients, 31 had primary infertility, while 52 had secondary infertility. The age of these patients ranged between 23-45 years old, with an average age of $31.4 \pm 5.7$ years old. The course of infertility ranged from 1-9 years, with an average of $3.9 \pm 2.1$ years. The selected infertility patients were examined at 3-7 days after the flow of the menstrual cycle, when they provided a signed informed consent and met the indications of the oviduct ultrasound contrast examination.

\section{TVS RT-3D-HyCoSy inspection methods}

The GE Voluson E8 ultrasonic diagnostic apparatus was used, and Bracco SonoVue was the contrast agent used. Five ml of normal saline was infused and oscillated to mix. Then, a 2-ml suspension was obtained, added with normal saline, and prepared into a 20-ml diluent for future use. An intramuscular injection of $0.5 \mathrm{mg}$ of atropine was given before radiography. After intrauterine catheterization, the real-time three-dimensional imaging mode was started, and the contrast medium was slowly and uniformly injected into the catheter. The distribution of the contrast medium in the uterus, oviduct, pelvic cavity, and surrounding areas of the nests was observed, and the images were preserved. Afterward, three- and two-dimensional radiography images and three-dimensional volume images of the uterus were acquired. Then, the preserved images were processed. According to the image results, the patients were classified into two types based on tubal patency: unobstructed and obstructed. All infertility patients underwent the gold standard laparoscopic dye study after TVS RT-3D-HyCoSy. Then, the accuracy and misdiagnosis rate of TVS RT-3D-HyCoSy in assessing tubal patency was drawn, and the misdiagnosed cases were analyzed.

\section{Statistical analysis}

Data were statistically analyzed using SPSS 19.0 statistical software. Measurement data were expressed as mean \pm standard deviation $(x \pm S D)$, and compared between different groups using the Chisquare test. $P<0.05$ was considered statistically significant. The consistency of the test results was analyzed using the Kappa value.

\section{RESULTS}

\section{TVS RT-3D-HyCoSy results}

Among these 83 infertility patients, 15 were diagnosed with patency of bilateral oviducts by TVS RT-3D-HyCoSy, 29 were diagnosed with obstruction of bilateral oviducts, 35 had patency in one side and obstruction on the other side (Figures 1A and 1B), and four patients had only one oviduct due to a previous operation for ectopic pregnancy, among which three oviducts were unobstructed, and one was obstructed. Therefore, among these 162 oviducts, 68 oviducts were unobstructed, and 94 were obstructed.

Comparison of the TVS RT-3D-HyCoSy and laparoscopic dye study results

With the results of the laparoscopic dye studies as the gold standard, the accuracy rate of TVS RT-3D-HyCoSy in diagnosing tubal patency was $88.9 \%(144 / 162)$, and the misdiagnosis rate was $11.1 \%$ (18/162). Furthermore, the sensitivity of diagnosing oviduct obstruction was $89.6 \%(86 / 96)$, the positive predictive value was $91.5 \%(86 / 94)$, the specificity of diagnosing tubal patency was $87.9 \%$ (58/66), and the negative predictive value was $85.3 \%(58 / 68)$. The accuracy of TVS RT-3D-HyCoSy was similar to that of these laparoscopic dye studies, the difference was not statistically significant, and the consistency between these two was good (Table 1).

TABLE 1. COMPARISON OF THE TVS RT-3D-HYCOSY AND LAPAROSCOPIC DYE STUDY RESULTS

\begin{tabular}{l|l|l|l}
\multirow{2}{*}{ TVS RT-3D-HyCoSy } & \multicolumn{2}{|l|}{ Laparoscopic } & Total \\
\cline { 2 - 4 } & unobstructed & obstructed & \\
\hline unobstructed & 58 & 10 & 68 \\
\hline obstructed & 8 & 86 & 94 \\
\hline Total & 66 & 96 & 162 \\
\hline Note: $P=0.815, \kappa=0.771$ & \multicolumn{2}{|l}{}
\end{tabular}

TABLE 2. CAUSES OF MISDIAGNOSIS

\begin{tabular}{l|l|l} 
Results & Causes & $\mathrm{n}$ \\
\hline \multirow{2}{*}{$\begin{array}{l}\text { False } \\
\text { positive }\end{array}$} & contrast medium countercurrent and diffusion & 2 \\
\cline { 2 - 3 } & oviduct spasm & 2 \\
\cline { 2 - 3 } & abnormal shape or position of the oviduct & 3 \\
\cline { 2 - 3 } & poor imaging operation & 1 \\
\hline \multirow{2}{*}{$\begin{array}{l}\text { False } \\
\text { negative }\end{array}$} & contrast medium countercurrent and diffusion & 4 \\
\cline { 2 - 3 } & oviduct hydrops & 2 \\
\cline { 2 - 3 } & $\begin{array}{l}\text { contralateral contrast medium diffused to the } \\
\text { affected side }\end{array}$ & 2 \\
\cline { 2 - 3 } & pelvic adhesion & 2 \\
\hline \multirow{2}{*}{ Total } & & 18 \\
\hline
\end{tabular}




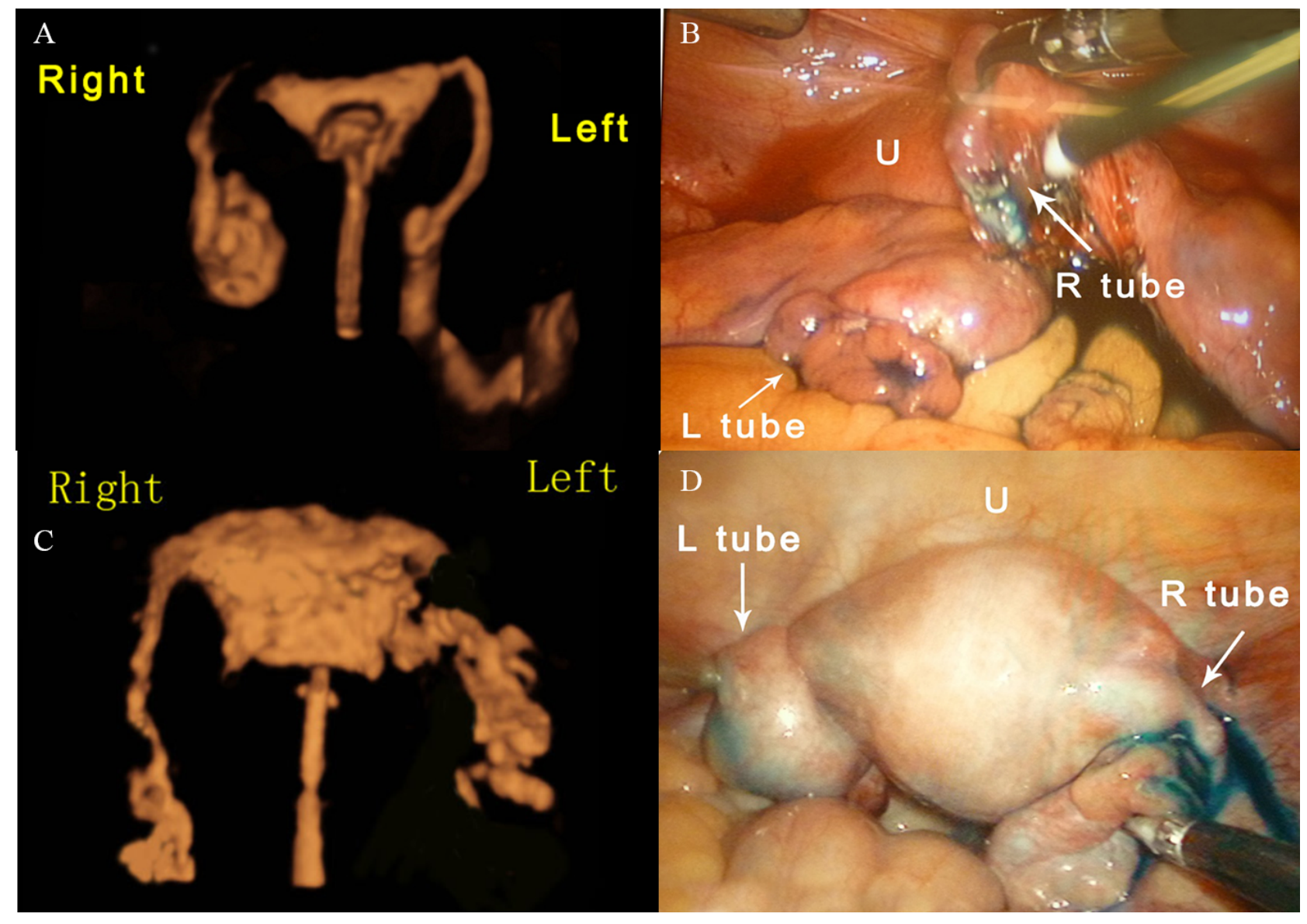

FIGURE 1A. TVS RT-3D-HyCoSy reveals that the left oviduct is unobstructed, and the right oviduct is obstructed.

FIGURE 1B. The laparoscopic surgery revealed that the left oviduct fimbria has an outflow of methylene blue, the right oviduct was conglutinated, and no outflow of methylene blue was found on the oviduct fimbria.

FIGURE 1C. The TVS RT-3D-HyCoSy revealed that that right oviduct is unobstructed, and contrast agent countercurrent was found on the left side of the uterus.

FIGURE 1D. The laparoscopic surgery revealed that the right oviduct fimbria had an outflow of methylene blue, while the left oviduct fimbria had no outflow of Meilan, but blue staining beside the uterus could be observed.

\section{Causes of misdiagnosis}

The TVS RT-3D-HyCoSy results were false negative in eight oviducts and false positive in 10 (Figures 1C and 1D). The specific reasons are presented in Table 2.

\section{DISCUSSION}

In the present study, the advantages of TVS RT-3D-HyCoSy were fully verified. This method is safe, accurate, simple, easy to operate, noninvasive, has no radiation or impact on pregnancy, is a new and ideal method to check tubal patency, and has been well accepted by doctors and patients. Furthermore, TVS RT-3D-HyCoSy is a better examination technique for infertile women in the field of assisted reproductive technology, even expected to replace traditional lipiodol radiography, and is suitable for wider clinical promotion. ${ }^{6}$ However, the present study revealed that approximately $30 \%$ of images acquired by TVS RT-3D-HyCoSy were poor in quality, and some of these even affected the analysis of results. Furthermore, the patency of $11.1 \%$ of oviducts (18 oviducts) was not consistent with the real results, causing excessive laparoscopic surgery induced by false-positive results, or delayed surgery and pregnancy induced by false-negative results. This brings some perplexity and interference in clinic practice.

TVS RT-3D-HyCoSy can automatically display the three-dimensional shape of the oviduct in real-time. The tubal patency is mainly determined by the shape of the oviducts after radiography. Indeed, it must be combined with other factors, including the resistance of the bolus injection of contrast agent and the situations of the outflow of the contrast agent from the oviduct fimbria, especially the contrast agent 
surrounding the ipsilateral ovary. ${ }^{7,8}$ Therefore, the quality of oviduct images by TVS RT-3D-HyCoSy is the key to determine tubal patency. ${ }^{9}$ The present study revealed that the quality of the image was closely correlated to the situation in the pelvic cavity of the patient. In patients with a history of pelvic or intrauterine surgery, patients with pelvic adhesions or pelvic endometriosis, and patients with secondary infertility, the proportion of poor image quality was relatively high. Among the causes of misdiagnosis, the proportion of countercurrent and diffusion of the contrast agent was the highest. The countercurrent and diffusion of the contrast agent would conceal the image of the oviduct. Furthermore, a small amount of concealment can be distinguished based on experience, while excessive interference of the contrast agent would make the image complex. Moreover, it would sometimes be difficult to find the true oviduct, or it would be difficult to determine whether there was any outflow of contrast agent from the oviduct. TVS RT-3D-HyCoSy can retain the whole video showing the moment when the contrast agent flows into the uterine cavity until it outward flows to the pelvic cavity. However, the countercurrent and dispersion of the contrast agents also appear in this process. Although it can be identified and excluded by playback, there is still a situation in which there is no way to distinguish the oviduct after the interference. Hence, the real oviduct could not be found, or the countercurrent of the contrast agent is regarded as an oviduct, leading to a false positive. A previous study revealed that the countercurrent is correlated to the thickness of the endometrium, the timing of radiography, and the history of pelvic operation. Choosing the right time for radiography and reducing the damage to the endometrium caused by radiography can reduce but not completely avoid the occurrence of countercurrent. ${ }^{10}$ If image interference is obvious, the pelvic cavity can also be scanned by energy or color Doppler ultrasound, in which injection imaging is performed again after blasting the contrast agent microbubbles. ${ }^{11}$

Abnormal shape or position of the oviduct itself is also a common cause of misdiagnosis. Pelvic adhesions can induce the oviduct to be lifted, or cause excessive distortion and a complex image of the oviduct. ${ }^{12}$ The oviduct is pulled by the lateral adhesion to change the original distributions, the outflow of contrast agent from the oviduct fimbria can flow to the periphery of the contralateral ovary, and adhesion can make the distal oviduct to go beyond the scope of scanning, making it difficult to distinguish whether the distal oviduct is unobstructed or obstructed. In a two-dimensional ultrasound examination, the topographical relationship of the ovary and uterus can roughly predict the distribution of the oviduct. The results of the present study revealed that when the ovary was tightly attached to the uterus, the oviduct would also be close to the uterus, making it difficult to identify. If the patient has uterine fibroids and ovarian cysts that pushes the oviduct, it can also change the normal distribution of the oviduct, making the image difficult to be recognized ${ }^{13}$. Furthermore, the outflow of the contrast agent from the oviduct fimbria is sometimes not distributed along the periphery of the ipsilateral ovary, affecting the judgment of the results. When the uterus is in its middle position, the proximal oviduct would be far in the three-dimensional space image, and the proximal oviduct would be thinner. Therefore, when the oviduct is in the middle position, the image of the proximal oviduct is not well-displayed; that is, the starting point is not clear. This affects the search for the distal oviduct, which can easily lead to errors. The median uterus can be turned into the anterior or posterior uterus by pressing the abdomen or shaking the probe, and an examination would be conducted again. When the oviduct is longer, the image of the outflow of the contrast agent from the distal oviduct is not collected, and it would be mistaken as an obstruction in the distal end. Therefore, in radiography, it is necessary to increase the acquisition frame appropriately and concomitantly keep both the uterine horn and bilateral ovary in the acquisition frame in order to allow the full length of the oviduct to be observed. When the oviduct presents hydrops, the accumulation of the contrast agent in the distal end would sometimes be regarded as an outflow of the contrast agent from the oviduct fimbria, causing a false negative. In patients with uterine malformation, especially those with complete double horns or mediastinum, the contrast tube can only be inserted into one side of the uterus. Furthermore, intrauterine adhesions make the uterine cavity narrow and even cause the opening of the oviduct to be blocked. This causes the contrast agent to fail to flow smoothly into the oviduct, leading to misdiagnosis. Therefore, attention should be given to the three-dimensional ultrasound examination of the uterine cavity shape. ${ }^{14}$

Unreasonable operation during radiography 
would also cause misdiagnosis. ${ }^{15}$ When the balloon is filled and becomes too large, or the position of the balloon is too high, the resistance of the bolus injection of contrast agent would be increased, and the contrast agent would not be able to flow outward smoothly. This situation would be mistaken as obstructed oviduct. When the contrast tube inserted into the uterine cavity is on the side of the uterine horn, there would be a difference in the time when the contrast agent flows outward from the bilateral oviducts. If the time interval is too long, it would be mistaken as an obstruction of the contralateral oviduct. The results of the present study revealed that these factors could be corrected by changing the size and location of the balloon. In radiography, attention should be given to the situation of the contrast agent reflux. If the inside of the cervix is loose, especially in the multipara, and when reflux occurs, intrauterine pressure would not be enough, and the amount of contrast agent entering into oviduct decreases. This would easily lead to a misdiagnosis. This can be corrected by tightening the contrast tube or enlarging the balloon, allowing it to be plugged to the cervical intraoral cavity. This way, it can allow the contrast agent to enter the oviduct smoothly, obtaining the correct results. The diameter of the contrast agent particles is equal to that of red blood cells, so a very fine oviduct can allow the contrast agent to enter. ${ }^{16}$ Therefore, when the internal oviduct is narrow, but not completely occluded, there would be a small amount of contrast agent entering the oviduct, and an unobstructed result may be obtained. However, in clinic practice, such oviducts are fine, stiff, and dys- functional. In addition, when oviduct spasm occurs, the contrast agent cannot temporarily pass through the oviduct, leading to a false-positive result. Spasms are correlated to tension or pain of the patient. ${ }^{17}$ In operation, the incidence of spasms can be reduced by soothing the patient or delaying the bolus injection of the contrast agent for a short period of time. Slight adhesion of the oviduct or the accumulation of endometrial debris at the opening of the oviduct may hinder the contrast agent from passing through at the beginning. The pressurizing bolus injection of the contrast agent can sometimes remove interference, making the oviduct unobstructed.

In summary, through the analysis of misdiagnosed cases, the researchers consider that some causes for misdiagnosis of TVS RT-3D-HyCoSy can be avoided, while some are very difficult to avoid. Patients with poor image quality still need doctors with some experience to process the images and determine the results. Furthermore, understanding and improving the cause of misdiagnosis can improve the diagnostic performance of TVS RT-3D-HyCoSy.

\section{Conflicts of interest}

All authors have contributed significantly to the manuscript and declare that the work is original and has not been submitted or published elsewhere. None of the authors have any financial disclosure or conflict of interest.

\section{Funding}

This study was supported by the Capital health development research project [No.2014-4-2115]

\section{RESUMO}

OBJETIVO: Este estudo tem como objetivo investigar as causas do diagnóstico equivocado na avaliação da patência tubária por meio da ultrassonografia de contraste histerosalpingo em tempo real transvaginal (TVS RT-3D-HyCoSy), a fim de melhorar a eficiência diagnóstica das TVS RT-3D-HyCoSy.

MÉTODOS: Um total de 162 ovidutos em 83 pacientes da infertilidade foi examinado por TVS RT-3D-HyCoSy. Esses resultados foram comparados com o padrão ouro para estudos de tintura laparoscópica, e os casos diagnosticados erroneamente foram analisados.

RESULTADOS: TVS RT-3D-HyCoSy revelaram que 68 ovidutos foram desobstruídos e 94 ovidutos foram obstruídos. Os resultados para os 144 ovidutos estavam em consonância com o padrão ouro, enquanto que aqueles para os 18 ovidutos, não. A taxa de acurácia do TVS RT-3D-HyCoSy foi de 88,9\%, e a taxa de erro de diagnóstico foi de 11,1\%. As principais causas de erro de diagnóstico incluíram contraponto e difusão do meio de contraste, espasmo do oviduto, forma ou posição anormal do oviduto, adesão pélvica e má operação de imagem.

CONCLUSÃO: TVS RT-3D-HyCoSy pode bem avaliar a patência tubária, e compreender e melhorar a causa do erro de diagnóstico. Além disso, a eficiência diagnóstica do TVS RT-3D-HyCoSy ainda pode ser melhorada.

PALAVRAS-CHAVE: Histerossalpingografia. Infertilidade. Técnicas de diagnóstico obstétrico e ginecológico. Erros de diagnóstico. 


\section{REFERENCES}

1. Lim CP, Hasafa Z, Bhattacharya S, Maheshwari A. Should a hysterosalpingogram be a first-line investigation to diagnose female tubal subfertility in the modern subfertility workup? Hum Reprod. 2011;26(5):967-71.

2. Exacoustos C, Di Giovanni A, Szabolcs B, Binder-Reisinger H, Gabardi C, Arduini D. Automated sonographic tubal patency evaluation with three-dimensional coded contrast imaging $(\mathrm{CCl})$ during hysterosalpingo-contrast sonography (HyCoSy). Ultrasound Obstet Gynecol. 2009;34(5):609-12.

3. Exacoustos $C$, Di Giovanni A Szabolcs B, Romeo V Romanini ME, Luciano $D$, et al. Automated three-dimensional coded contrast imaging hysterosalpingo-contrast sonography: feasibility in office tubal patency testing. Ultrasound Obstet Gynecol. 2013;41(3):328-35.

4. Serra V, Olmo I, Albert MM, Lara C, Remohi I, Pellicer A. An audit of our experience on the use of hysterosalpingo-contrast-sonography (HyCoSy). Ultrasound Obstet Gynecol. 2017;50(suppl. 1):386-7.

5. Shahid N, Ahluwalia A, Briggs S, Gupta S. An audit of patients investigated by Hysterosalpingo-Contrast-Sonography (HyCoSy) for infertility. J Obstet Gynaecol. 2005;25(3):275-8.

6. Ludwin I, Ludwin A, Nastri CO, Coelho Neto MA, Kottner J, Martins WP. Evaluating tubal patency using three different ultrasound techniques: interobserver reliability, agreement and diagnostic accuracy. Ultrasound Obstet Gynecol. 2017;50:240.

7. Zhou L, Zhang $X$, Chen X, Liao L, Pan R, Zhou N, et al. Value of three-dimensional hysterosalpingo-contrast sonography with SonoVue in the assessment of tubal patency. Ultrasound Obstet Gynecol. 2012;40(1):93-8.

8. Wang W, Zhou Q, Gong Y, Li Y, Huang Y, Chen Z. Assessment of fallopian tube fimbria patency with 4-dimensional hysterosalpingo-contrast sonography in infertile women. J Ultrasound Med. 2017;36(10):2061-9.

9. He Y, Geng Q, Liu H, Han X. First experience using 4-dimensional hysterosalpingo- contrast sonography with SonoVue for assessing fallopian tube patency. J Ultrasound Med. 2013;32(7):1233-43.
10. Giugliano E, Cagnazzo E, Bazzan E, Patella A, Marci R. Hysterosalpingo-contrast sonography: is possible to quantify the therapeutic effect of a diagnostic test? Clin Exp Reprod Med. 2012;39(4):161-5.

11. Marci R, Marcucci I, Marcucci AA, Pacini N, Salacone P, Sebastianelli A, et al. Hysterosalpingocontrast sonography (HyCoSy): evaluation of the pain perception, side effects and complications. BMC Med Imaging. 2013;13:28.

12. Hong Q, Cai R, Chen Q, Zhang S, Ai A, Fu Y, et al. Three-dimensional HyCoSy with perfluoropropane-albumin microspheres as contrast agents and normal saline injections into the pelvic cavity for morphological assessment of the fallopian tube in infertile women. J Ultrasound Med. 2017;36(4):741-8

13. Kong D, Dong $X$, Wang $Z$, Zhang L, Shao X, Qi Y. Four-dimensional hysterosalpingo-contrast sonography with auxiliary hydrogen peroxide examination for the diagnosis of fallopian tube patency following interventional treatment of ovarian ectopic cysts. Arch Gynecol Obstet. 2017;295(2):519-26.

14. Piccioni MG, Riganelli L, Filippi V, Fuggetta E, Colagiovanni V, Imperiale L, et al. Sonohysterosalpingography: comparison of foam and saline solution. J Clin Ultrasoud. 2017;45(2):67-71.

15. Dreyer K, Out R, Hompes PG, Mijatovic V. Hysterosalpingo-foam sonography, a less painful procedure for tubal patency testing during fertility workup compared with (serial) hysterosalpingography: a randomized controlled trial. Fertil Steril. 2014;102(3):821-5.

16. Chegondi N, Gopi S. HyCoSy by using the new ultrasound contrast agent lignosal (mixture of $2 \%$ lignocaine jelly and saline) for the evaluation of tubal patency. Ultrasound Obstet Gynecol. 2017;50(suppl. 1):56.

17. Moro F, Selvaggi L, Sagnella F, Morciano A, Martinez D, Gangale MF, et al. Could antispasmodic drug reduce pain during hysterosalpingo-contrast sonography (HyCoSy) in infertile patients? A randomized double-blind clinical trial. Ultrasound Obstet Gynecol. 2012;39(3):260-5. 
Erratum

http://dx.doi.org/10.1590/1806-9282.65.12.1515

Regarding the article "Causes of misdiagnosis in assessing tubal patency by transvaginal real-time three-dimensional hysterosalpingo-contrast sonography", with DOI number: http://dx.doi. org/10.1590/1806-9282.65.8.1055, published in Journal of the Brazilian Medical Association, 2019;65;08, page 1055

Affiliation number 1 must be changed from: Department of Ultrasound, Beijing Obstetrics and Gynecology Hospital of Capital Medical University, Beijing 100026, China

Now read: Department of Ultrasound, Beijing Obstetrics and Gynecology Hospital, Capital Medical University, Beijing 100026, China 